\title{
In-Situ Catalysis with High Spatial and Temporal Resolution
}

\author{
J.T. McKeown, ${ }^{*}$ S. Mehraeen, ${ }^{* *}$ D.J. Masiel, ${ }^{* * *}$ B.W. Reed, ${ }^{*}$ T.B. LaGrange, ${ }^{*}$ J.E. Evans, ${ }^{* *}$ \\ G.H. Campbell,* and N.D. Browning******* \\ * Condensed Matter and Materials Division, Lawrence Livermore National Laboratory, Livermore, \\ CA 94550 \\ ** Department of Molecular and Cell Biology, University of California, Davis, CA 95616 \\ *** Department of Chemical Engineering and Materials Science, University of California, Davis, \\ CA 95616
}

An in-situ characterization technique capable of imaging at both high spatial and temporal resolution can provide a complete understanding of the mechanisms of catalytic processes by allowing analysis of gas-solid interactions at ambient pressures and elevated temperatures prior to, during, and after a reaction. The dynamic transmission electron microscope (DTEM) provides this ability to study catalysis in-situ with nanometer spatial resolution and nanosecond time resolution [1,2], generating several orders of magnitude increase in spatio-temporal resolution over conventional video frame rates. The current DTEM uses electron pulses $\sim 15 \mathrm{~ns}$ in duration for single-shot imaging of dynamic processes, which minimizes electron-beam interactions during dynamic studies. The next-generation DTEM, which is currently being installed, will be capable of $\mu$ s electron pulses that can provide atomic-scale spatial resolution for imaging.

The use of the DTEM to study supported nanoscale metal particles and clusters is focusing on the identification and control of active sites on catalyst nanoparticles, which are responsible for the selectivity of chemical reactions and nucleation of nanostructures. Gaseous environments that cannot be obtained in the electron microscope are achieved using in-situ gas stages that incorporate a windowed-cell design into the TEM holder. Gas stages have been designed and built for both the DTEM and an aberration-corrected TEM. The stage for the aberration-corrected TEM uses a continuous-wave laser built in to the holder for heating of the specimen while the DTEM uses a pulsed laser for ultrafast laser heating. Reactions can be controlled by the gas partial pressure within the stage to ensure that interactions occur on the desired timescale for imaging or diffraction. This combination of in-situ DTEM and in-situ aberration-corrected TEM allows studies of catalytic reactions across multiple spatial and time scales.

The results of initial oxidation/reduction experiments of thin-film specimens will be presented along with preliminary results on catalyst nanoparticles and clusters. Figure 1 shows an example image of a cluster of $\mathrm{Co}_{3} \mathrm{O}_{4}$ particles with the associated diffraction patterns, obtained in the DTEM gas stage. There is an increase in diffuse scattering with gas pressure, as expected and evident from the diffraction patterns, but minimal loss of resolution in the images. All aspects of the design, implementation, and characterization of the in-situ gas stages within the DTEM platform will also be discussed in this presentation [3].

\section{References}

[1] W.E. King et al., J. Appl. Phys. 97 (2005) 111101-1.

[2] T.B. LaGrange et al., Ultramicroscopy 108 (2008) 1441. 
[3] This work was performed under the auspices of the U.S. Department of Energy by Lawrence Livermore National Laboratory and supported by the Office of Science, Office of Basic Energy Sciences, Division of Materials Sciences and Engineering, of the U.S. Department of Energy under Contract No. DE-AC52-07NA27344. Development of in-situ stages at UC Davis was supported by DOE NNSA-SSAA grant number DE-FG52-06NA26213 and NIH grant number RR025032-01.
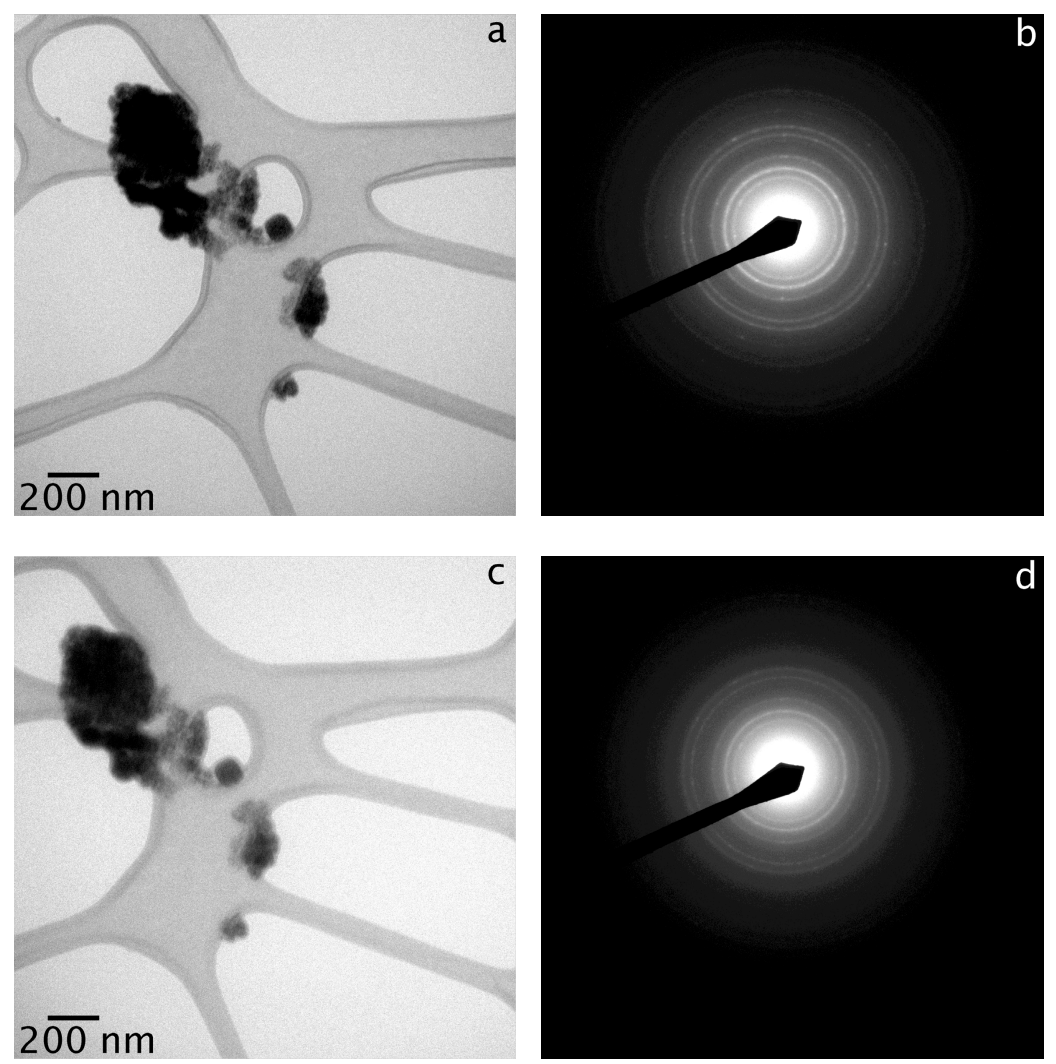

FIG. 1. a) Bright-field TEM image of a cluster of $\mathrm{Co}_{3} \mathrm{O}_{4}$ nanoparticles on a carbon support with b) the associated diffraction pattern, obtained in the DTEM gas stage at a pressure of $\sim 2$ Torr. When the pressure is increased to $\sim 340$ Torr, c) there is a minimal loss of resolution in the bright-field image as a result of an increase in diffuse scattering, evident in d) the diffraction pattern. 